tendency of such solutions to show a lowered oxidizing capacity after standing. It occurred to the writers that this might be due to a slow reaction with traces of hydrogen peroxide which had not been decomposed by boiling the alkaline solution. To test this point, the solution obtained by dissolving the fused mass from a sample of ferrochromium was boiled $30 \mathrm{~min}$., made up to volume, and on analysis found to contain 63.42 per cent chromium. After 4 days the apparent percentage of chromium was 62.10 and 62.20 . A solution of another fusion was boiled $2 \mathrm{hrs}$. before acidification. The analysis of the freshly prepared solution gave
63.53 per cent chromium. After 4 days the apparent percentage of chromium was 63.05 and 63.15 per cent. It is thus clear that even boiling 2 hrs. does not give a stable solution.

\section{Summary}

In this paper a method has been described for determining chromium in ferrochromium in which the chromate produced by oxidizing either with sodium peroxide or with silver nitrate and ammonium persulfate is titrated with ferrous sulfate, using an electrometric end-point. The procedure described has been in use in this laboratory for several years.

\title{
The Use of Perchloric Acid as an Aid to Digestion in the Kjeldahl Nitrogen Determination
}

\author{
By Brainerd Mears and Robert E. Hussey \\ Thompson Chemical Laboratory, Williams Colifigh, Wilinamstown, Masgachusatto
}

On observing the corrosive action of 60 per cent perchloric acid on organic matter at somewhat elevated temperatures, it was decided to carry out a series of experiments in which small quantities of this material were used as an aid to digestion in the Kjeldahl determination of nitrogen. It was feared that the combination of sulfuric acid, perchloric acid, and organic matter might lead to serious explosions, but after running over 500 determinations with varying additions of perchloric acid without an explosion and at most with only a rapid deflagration, it was determined that this reaction was not particularly dangerous. In most instances, however, the perchloric acid, sulfuric acid, and other materials were mixed cold. It was also feared that there might be considerable loss of nitrogen due to the volatilization or decomposition of the ammonium perchlorate which might be formed at the elevated temperatures, or that other interfering factors might develop.

\section{Experimental Details}

A series of preliminary experiments proved that the addition of copper sulfate was beneficial, that it was impracticable to attempt to regulate the temperature of the digestion, and that most of the ammonia remained in the digestion flask. Accordingly, with a view to obtaining comparable data on the use of the perchloric acid, the following experiments were carried out. One-gram samples of casein, tankage, egg albumin, gelatin, and dried blood, and about 10-g. samples of urine and milk were treated with 25 cc. of concentrated sulfuric acid and $1 \mathrm{~g}$. of copper sulfate. Several determinations were run by the official Gunning method, slightly modified, ${ }^{2}$ as a check on the nitrogen content of the material and to furnish an estimate of the time required for the complete digestion of the sample. At the same time, a series of determinations containing the same quantities of sulfuric acid and copper sulfate but with increasing amounts of the perchloric acid was carried on. Blank checks were run on the reagents in each instance, the average of which showed a nitrogen content of 0.0027 per cent. In some instances, because of the lack of uniformity or low nitrogen content of the samples, the accuracy of the determinations might have been increased if larger samples had been employed, but it was considered advisable to keep the quantity of the sample constant to assist in drawing conclusions from the results obtained. The $1 \mathrm{~g}$. of copper sulfate employed was probably more than was required; but inasmuch as many of the experiments were run with this quantity and no harmful effects were observed from its presence, the same quantity was employed in all the determinations. The study of the Kjeldahl method by the Association of Official

1 Received June 3, 1921.

2 A. O. A. C., "Methođs of Analysis," 1920, 7.
Agricultural Chemists, ${ }^{1}$ partly substantiates these conclusions. Table I summarizes the results obtained.

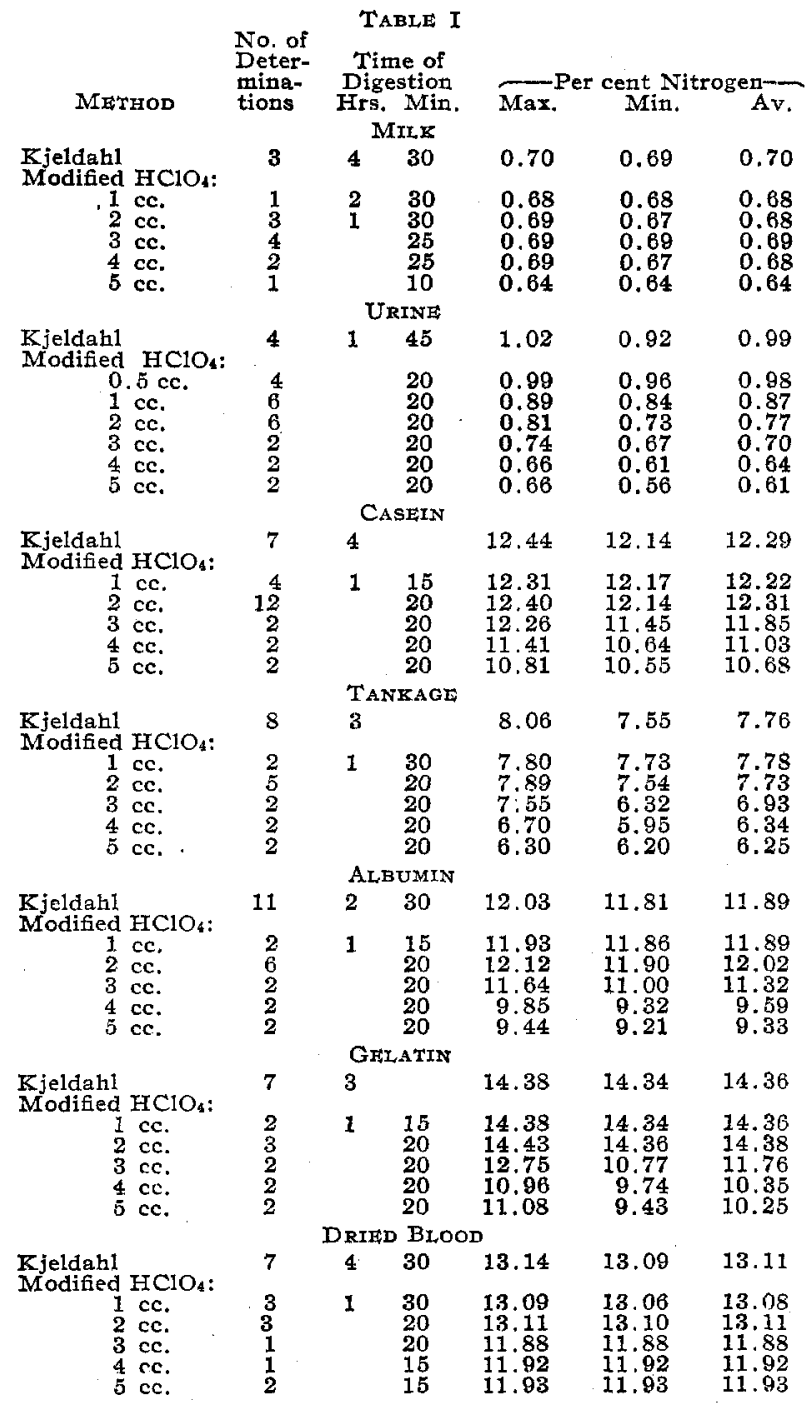

The curves which follow were obtained by employing the above data and plotting the percentage of nitrogen against the quantities of perchloric acid used.

\section{Discussion of Results}

It is to be observed that, with the constant condition of a 1-g. sample, plus $1 \mathrm{~g}$. of copper sulfate and $25 \mathrm{cc}$. of con-

1 Vol. 4, 367. 


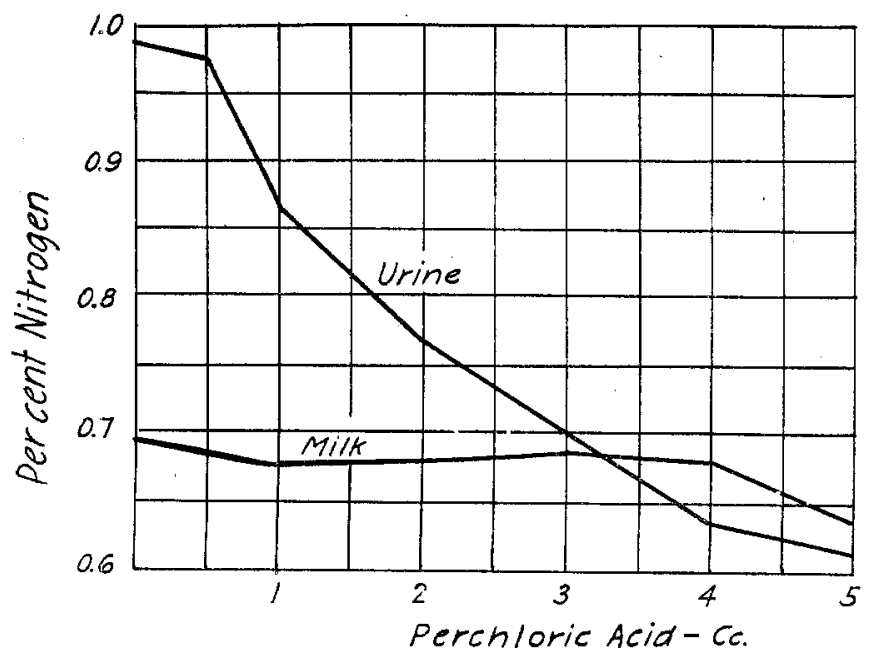

centrated sulfuric acid, the per cent of nitrogen obtained remains fairly constant, with a marked decrease in the time required for the digestion, up to the addition of $2 \mathrm{cc}$. of the perchloric acid to the $1 \mathrm{~g}$. of solid material. Beyond that point there is a rapid and increasing loss of nitrogen, as shown by the slope of the curves. The plotted results of the milk and urine show curves which differ somewhat from the solid samples, but here the presence of water and the varying quantity of solid material, although they change conditions to some extent, apparently point to relatively the same conclusions.

Confining our observations to the solids analyzed, we conclude that 2 cc. of 60 per cent perchloric acid per gram of solid material furnishes the most rapid digestion and gives results which are influenced little, if at all, by the presence of this acid. It was also observed that the best perchloric acid results were obtained from determinations which cleared in not less than $3 \mathrm{~min}$. or more than $7 \mathrm{~min}$. from the time of lighting the gas under the digestion flask, and which were heated for $15 \mathrm{~min}$. after clearing. The $2 \mathrm{cc}$. of perchloric acid and the 1-g. sample usually conformed to these requirements. It would seem then that sufficient perchloric acid must be present to decompose the organic material, but that the presence of an excess causes a loss of nitrogen.

Experiments performed on 2-g. samples of the solid material with $4 \mathrm{cc}$. of perchloric acid gave comparable results. Attempts were also made to add an excess of perchloric acid, digest and clear the sample, and neutralize the undesired excess of acid by the addition of pure cane sugar. The results were of fair accuracy, but flaming and other attendant difficulties caused the procedure to be abandoned. The use of perchloric acid, diluted with water, and the use of smaller quantities of sulfuric acid, even as little as $10 \mathrm{cc}$. in some instances, gave accurate results; but in many cases there was a considerable loss of nitrogen.

Experiments were also carried on by adding perchloric acid in varying quantities in the determination of nitrogen in nitrates by the Gunning method. While results of fair accuray were obtained, little time was saved because of the time required for the cooling of the digestions before the addition of the acid, and it was therefore concluded that little was to be gained by the application of the procedure to this class of substances, except perhaps where the substances employed were exceedingly difficult to digest.

\section{Application of Method to Other Stubstances}

In order to apply the method to a greater variety of substances, samples of sheep manure, cocoa shells, castor pumace, rapeseed meal, and hoof meal of known nitrogen content were obtained from a most reliable source. Many of the samples were reported to be difficult to digest and in some instances had lost moisture on storage. Some samples were not uniform in composition.

By the use of 1-g. samples with $25 \mathrm{cc}$. of concentrated sulfuric acid, $1 \mathrm{~g}$. of copper sulfate, and $2 \mathrm{cc}$. of 60 per cent perchloric acid (unless the trial run showed by its rate of clearing a need of more or less of this acid) the results recorded. in Table II were obtained. It seems that they may be considered a fair check when obtained by two independent investigators working in different laboratories at different times.

TABLE II

(Time of digestion, 20 min.)

No. of

Deter-

Deter-

Substance

Sheep manure

Cocoa shells 1

Castor pomace

Raptor pomace.

Garbage tankage

Cattle manure

Tankage $(6-30)^{1}$

Tankage $(10-20)$

(dark)

Hoof meal 1

tions

6

II

Maximue cent Nitrogen-

Per cent

Stated to Be

5
5
3

5
3
3

5

4
4
3
3

1 Corrected for moisture.

23 cc. of perchloric acid used.

$\begin{array}{lll}1.54 & 1.41 & 1.48 \\ 2.43 & 2.41 & 2.42 \\ 5.83 & 5.67 & 5.72 \\ 3.54 & 3.37 & 3.45 \\ 5.69 & 5.67 & 5.68 \\ 8.44 & 8.41 & 8.42 \\ 2.17 & 1.92 & 2.09 \\ 4.86 & 4.73 & 4.81 \\ 7.67 & 7.50 & 7.61 \\ 6.59 & 6.48 & 6.54\end{array}$

$\begin{array}{rrr}6.59 & 6.48 & 6.54 \\ 15.35 & 15.27 & 15.32\end{array}$ Present in

1.48

1.48
2.22

2.22

3.33

3.33
5.74

8.58

1.88

4.98
7.62

6.69

15.23

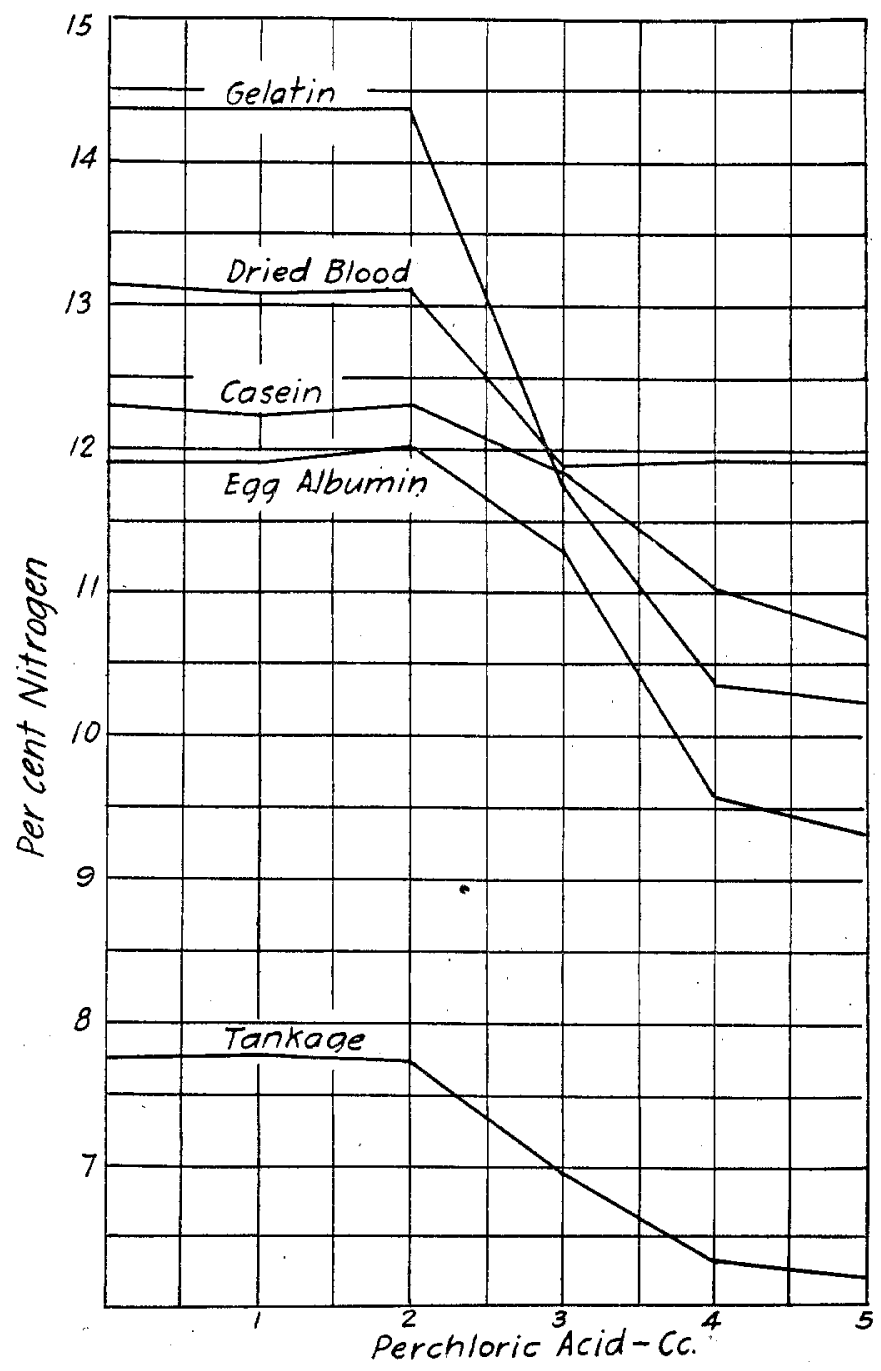


SUMMARY

From the above results it is concluded that the use of small quantities of 60 per cent perchloric acid considerably shortens the time of digestion in a Kjeldahl determination, without appreciably affecting the accuracy of the results, in the class of substances investigated, provided that the quantity of the acid added does not cause the digestion to clear in less than $3 \mathrm{~min}$. or fail to clear in $7 \mathrm{~min}$.; that $1-\mathrm{g}$. samples treated with $25 \mathrm{cc}$. of concentrated sulfuric acid plus $1 \mathrm{~g}$. of copper sulfate with $2 \mathrm{cc}$. of perchloric acid usually fulfil these requirements; and that all samples should be heated at least. 15 min. after clearing.

\title{
LABORATORY AND PLANT
}

\section{Constant Temperature Rooms ${ }^{1,2}$}

\author{
By E. G. Hastings
}

Laboratories of the Department of Agricultural Bactertology, University of Wisconsin, Madison, Wisconsin

Until comparatively recently the constant temperature room was considered an essential part of the equipment of a bacteriological laboratory, but not of a chemical laboratory. With the widening interest and activity in biochemistry, the chemist has come to consider such a room as a vital portion of his equipment. In many, indeed in most cases, a large amount of money has been spent in providing complicated heating devices and in insulating the rooms.

The incubator equipment of the Department of Agricultural Bacterjology of the University of Wisconsin has been gradually developed, as the need for increased space has arisen. The need has been met with limited resources. It is thought that a brief presentation of the essential portions of the equipment may prove of interest to others who wish to extend their facilities in this direction.

The laboratories are provided with four incubator rooms of varying sizes. In each instance a room originally used as a store room and opening off a laboratory has been adapted for incubator purposes. No money has been spent in insulating the walls, the lath and plaster of the original building forming the only insulation. False ceilings have been installed in order to diminish the height of the rooms and to avoid heating space that was inaccessible. Two of the rooms are provided with double doors, one swinging into the room, the other outward. The other rooms have single, light wood doors.

\section{Heating Devices}

Three of the rooms are heated with gas. A bottomless box of galvanized iron (Fig. 1) about 16 in. $\times 14$ in. $\times 17$ in. high has been constructed with a door on one side. A row of holes near the bottom of the box permits the entrance of air. A rectangular galvanized iron pipe 12 by 6.5 in. in section is carried from the side of the box across one side of the room and then upward to the ceiling, where it joins a 6-in. round pipe which enters one of the ventilating flues of the building. A damper is installed in the round pipe to prevent the too rapid passage of air through the pipe and the consequent excessive loss of heat.

Gas is piped to the box and on the end of the supply pipe a rose-top Bunsen burner is connected with threaded joints. In the line of the supply pipe a Roux bimetallic regulator with a horseshoe shaped bar is installed with a bypass provided with a stopcock. The stopcock is for the purpose of allowing a small amount of gas to pass to the burner in case the regulator should not function. As far as our experience goes it is not an essential part of the equipment. As the heated gases pass through the pipe they are robbed of their heat by the extensive radiating surface of the pipe. The maximum use of the gas is thus obtained. One room has a content of $430 \mathrm{cu}$. $\mathrm{ft}$. The radiating surface of the box and

1 Received May 11, 1921.

2 Published with the permission of the Director of the Wisconsin Agricultural Experiment Station. pipe is approximately $52 \mathrm{sq}$. ft. The room is heated to a temperature of $35^{\circ} \mathrm{C}$. by one burner. The records presented in Fig. 2 show the temperature variations of the room during a period when it was opened only a few times each day. The maximum temperature variation of the rooms surrounding the room in question during the period covered by the records was $15^{\circ} \mathrm{C}$.

The fire hazard is nil, since the two possible sources of danger are excluded. Danger from a burner that strikes back is avoided by usins only threaded joints and by not allowing the supply pipe to come in contact with wood for 3 or $4 \mathrm{ft}$. from the burner. The danger from an extinguished burner is avoided by the passage of the gas into the ventilating flue. One of the rooms is so situated that a.ccess to 2

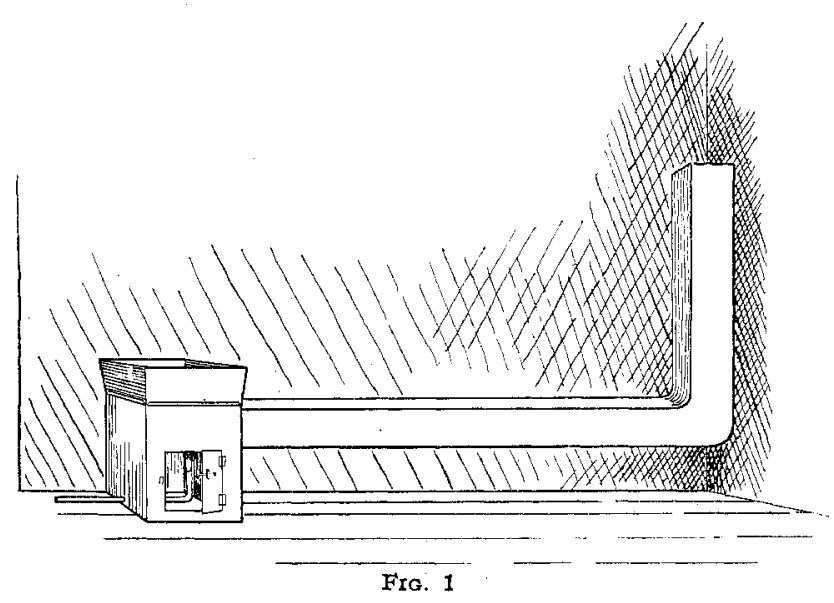

ventilating flue could not be gained. The gases from the heating pipe are discharged into a hall, the cubical content of which is so great that an explosive mixture would not be obtained, unless the gas was allowed to escape for many hours. The absence of danger from fire was well shown by an incident of recent occurrence. One of the rooms is provided with two burners. During the summer one is sufficient to maintain the temperature at the desired point, but in the winter two are needed. The gas was turned off the building for the purpose of making repairs. When it was turned on again, one of the burners was lighted, but not the other, which was discharging gas. This unlighted burner was not discovered for 48 hrs. The gas from it did not catch fire, although the two burners were in the same box and not over 4 in. apart. The current of air through the box was sufficient to prevent the ignition of the gas and to carry it away so rapidly as to prevent an explosive mixture being reached in the box. No odor of gas could be noted in the room. The unlighted condition of the one burner was detected by the lowered temperature of the room. 